\title{
Genome Editing and its Necessity in Agriculture
}

\author{
Asma Majid ${ }^{1}$, G.A. Parray ${ }^{2}$, Shabir H. Wani ${ }^{2}$, Mojtoba Kordostami ${ }^{3}$, \\ N.R. Sofi ${ }^{2}$, Showkat A. Waza ${ }^{2}$, A.B. Shikari ${ }^{2}$ and Shazia Gulzar ${ }^{1}$ \\ ${ }^{1}$ Division of Genetics and Plant Breeding, FoA, Wadura, Sopore -193201, SKUAST-K, \\ Jammu and Kashmir, India \\ ${ }^{2}$ Mountain Research Centre for Field Crops, Khudwani-192102, SKUAST-K, \\ Jammu and Kashmir, India \\ ${ }^{3}$ Faculty of Agricultural Sciences University of Guilan Rasht, Iran \\ *Corresponding author
}

\section{A B S T R A C T}

\begin{tabular}{|l|}
\hline K e y w o r d s \\
Genome editing, \\
DNA, Protein, \\
Transcription. \\
\hline Article Info \\
\hline Accepted: \\
31 September 2017 \\
Available Online: \\
10 November 2017
\end{tabular}

Genome editing is a technique in which changes are made in the DNA of any organism. A nuclease promotes breaks in the DNA at a specific sequence which is repaired by several mechanisms. It is one of the efficient technologies which enable us to change and edit genome of any organism precisely and accurately. Multiple genome editing technologies have been employed, including zinc finger nucleases, mega nucleases, clustered regularly interspaced short palindromic repeats (CRISPR) along with Cas9 protein and transcription activator-like effector nucleases to modify genome of an organism. These genome editing technologies exercise several repair systems in which through the applying site-specific nucleases, areas are altered. SSNs induce double-strand breaks (DSBs) at predetermined locus in the targeted genome, which can be repaired utilizing well-known procedures such as non-homologous end-joining or homology-directed repair. Genome editing technique is reliable for enhancing average yield to fulfil the booming demands of the world's current food shortage and to establish a viable and ecologically safe agriculture scheme, to more precise, productive, economical and eco-friendly.

\section{Introduction}

Genome editing is a technique in which DNA is inserted, deleted or replaced in a genome of any organism using genome editing tools. It could be used vastly to edit genome of any organism. By modifying genome of an organism we can manipulate the crop growth features in accordance with our major purposes as are increasing the production, eliminating the unfavourable traits and improving its resistance to various biotic and abiotic stressors. Although GM crops have achieved great success in supplementing crop breeding, but this technique confronts some technical challenges as it's expensive and due to possible unpredictable negative impacts on environment and human food safety concerns as well, opposition against them grows exponentially. Further, plant breeders are frequently employing mutation breeding using mutant generators, radiation (gamma rays or fast neutron) or chemical (ethyl methane sulfonate or EMS) treatment to accelerate crop improvement process. This mutagenic breeding technique expose plants 
to various mutagenic agents that are causing damage to the plant cells. During the natural DNA repair process, genetic changes such as mutations are introduced into the genetic makeup of plants including genes, these genetic altercations are random across the whole genome which result rarely positive outcomes but more often negative, further positive outcomes can be strongly linked with negative outcomes, moreover, natural mutations recurrence possibility is minute. Lately, advances in DNA sequencing technologies, in the respect of cost-effective, assist conducting elaborate evaluation of whole living organisms' genomes which led to a burst in our understanding of genomics (Carroll, 2014).Therefore, utilizing genome editing technology in a highly accurate and authentic fashion to change and improve the genome of any organism can be convincingly accomplished.

\section{Genome editing mechanisms}

Genome engineering is interceded by site specific nucleases that depend on creation of endonucleases able to engender double stranded breaks in a targeted genome array. SSN acquire a DNA-binding domain that binds to the target sequence (Gaj et al., 2013). The considered array divided by the site specific nuclease, that trigger a number of DNA repair processes at the targeted locus ranging from deletion to insertion of transgenes. These mechanisms involve nonhomologous end-joining (NHEJ) in which two DNA ends ligate together causing erasure or insertions (InDel) at the break site where DNA sequences ligates together, thus resulting in frame shift mutation which ultimately create a gene knockout. Another mechanism is homologous recombination (HR), in which both site specific nucleases and a DNA repair, arrange array correspondence to the introduced break site. DNA ends are ligated to the introduced pattern which results in gene insertion. The genome editing replication is depend on large and influential factors such as stage of cell cycle, species, tissue type and the applied frame for editing (Fig. 1).

\section{Genome editing tools}

\section{Mega nucleases}

Mega nucleases first identified in 80s, they target large DNA sequences of about 12 to 40 base pairs long which lead them to be highly specific in the utmost of genomes (Gallagher et al., 2014). Sequence of this size occurs generally once in an entire genome which make them exclusive tools for genome engineering, since naturally occurring mega nucleases are uncommon and inadequate to be used in genome editing, companies were manipulated mega nucleases in order to be utilized in genome editing. They are also called homing endonucleases. Once the DNA is broken, natural DNA repair processes in the cells initiated which allowing the insertion of a justified DNA array. DNA binding domain and catalytic domain in mega nuclease are linked so its construction is either expensive or labour-intensive as compared with other genome editing tools. Therefore, mega nucleases have major drawbacks which lead them to have a considerably low priority as option amongst genome engineering tools to work with.

\section{Zinc finger nucleases}

Zinc finger nucleases are an artificial genome editing tool which is constructed by fusing Zinc finger DNA binding domain with catalytic domain. Zinc finger nucleases consists of zinc finger proteins which in the interest of targeting a particular DNA array to generate double-stranded breaks by fusing it with non-specific Fok1 endonuclease they were manipulated. By taking advantage of DNA repair mechanism these nucleases can be used to alter the genome of any organisms 
which is mediated by targeting specific sequence and inducing break via nonspecific endonuclease, any zinc finger protein identifies 3 nucleotides in the target array. The break induced by zinc finger nuclease is mediated by site specific nuclease which not only by non-homologous end joining but homologous recombination, repair is feasible. Construction of zinc finger nucleases is difficult as compared to TALENs and CRISPR/CAS systems. Further this technique induces more off target effect in contrast with other genome editing techniques (Table 1).

\section{Transcription activator-like effector nucleases}

Transcription activator-like effector nucleases (TALENs) were named as a method of year by nature methods in 2011 (Baker \& Becker 2012). TALENs compose of transcription activator-like effectors (TALEs) fused with the non-specific Fok1 endonuclease naturally found in Flavobacterium okenkoides These TALEs proteins are naturally exuded by a bacteria, Xanthomonas spp, which gets bind to the targeted DNA sequence with the help of DNA-binding domain. Each duplication identifies a single nucleotide in target DNA. TALE protein comprises of $\mathrm{N}$ terminal domain, central repetitive regions and the $\mathrm{C}$ terminal domain. Middle repetitive regions consists of 34 amino acids which are identical to each other except for two amino acids at situations12 and 13 called as Repeat Variable di-residues (RVD) that determines specificity of TALEs repeat. Continuous thrust for the precise, advanced and easier tools resulted in the development of CRISPR/Cas9.

\section{CRISPR/Cas technology}

Clustered regularly interspaced short palindromic repeat/Cas9 system has been initially detected in bacteria as a defensive mechanism versus exterior DNA attack as bacteriophage. The CRISPR/Cas system consists of CRISPR RNA (crRNA) and transactivating crRNA (tracrRNA) associated with a Cas9 endonuclease. CRISPR, i.e., Clustered Regularly Interspaced Short Palindromic Repeats (CRISPRs) consists of a tandem direct repeat sequences followed by proto spacers, i.e. the spaces between these repeat sequences, both of which are derived from the invading elements (Kim and Kim, 2014). Scientists have engineered the two RNA sequences i.e. crRNA and tracrRNA into one guide RNA, which is followed by Protospacer Adjacent Motif (PAM) i.e., a 5'-NGG sequence. The sgRNA has 20 nucleotides at the 5'end that directs Cas9 to the complementary target site. The Cas 9 protein is an endonuclease which creates doublestranded breaks at the target site. This innovating mechanism of the CRISPR/Cas system replaced all other mechanisms of genome editing tools in the year 2012 known as RNA-guided engineered nucleases.

\section{Cas9 nucleases types}

\section{Native Cas9}

The double stranded breaks constructed by native Cas9 which is either by nonhomologous end joining or homologous directed repair can be repairable.

\section{Cas9 Nickase}

It's developed through mutation in native Cas9 which induces nicks instead of double strand breaks. Two Cas9 nickase can be used simultaneously. This system reduces the offtarget effects.

\section{Inactive dCas9}

The nuclease deficient catalytically inactive mutant version of Cas9 (dCas9) has been applied for RNA-guided transcription regulation, as a substitution for genome engineering (Gilbert et al., 2013; Qi et al., 
2013). Asan adjusted technique, frequently and in fact successfully has been employed for CRISPR interference (CRISPRi) and CRISPR activator (CRISPRa) as well as for remarkably effective and precise gene silencing and activation, accordingly, adoptingdCas9 with an effector and a sgRNA.

\section{Guide RNAs types}

\section{Truncated guide RNAs (truRNA)}

They are types of guide RNA with shorter region of target complementaries which increases specificity of Cas9 endonuclease.

\section{Ribozyme-gRNA-Ribozyme (RGR)}

These are synthetic genes which give rise to RNA molecule with ribozyme sequence, owing to their crave to produce considered guide RNA both in vivo and in vitro, they might be subjected to self- catalysed break.

\section{Polycistronic tRNA-gRNA (PTG/Cas9)}

It's tandemly arrayed tRNA-gRNA which are cleaved by tRNA processing system and targets various locations simultaneously.

\section{CRISPR interference in plants}

The CRISPRi has been superb instance of aRNA-guided, consistent and extremely effective modulation of the target genes transcription in plants by fusion of inactivated dCas9 to effector domains (Larson et al., 2013). Majorly, this approach utilizes for transcription adjustment as well as gene expression, however, recently found alternative applications in biology, CRISPR activator (CRISPRa) applied for gene activation. CRISPRi and CRISPRa libraries are capable to be applying as versatile tools to survey the complicated stress-driven characteristics in plant to conduct functional genomic analysis. Gilbert et al., (2015) recognized that the required target site for effective CRISPRi, comparative to the transcription start site of a specific gene, should lie from -50 to +300 base pairs.

\section{Application of genome editing techniques in crop improvement}

\section{Blast resistance in rice}

Various genome editing techniques including TALENS and CRISPR/CAS systems abundantly have been employed to promote disease resistance in rice. The main purpose to develop TALEN technology in rice was its potential applications inbreeding of diseaseresistant rice varieties. Rice blast disease occurs due to the interactions within the TAL effectors from the bacterial parasite Xanthomonas oryzae $p v$. oryzae and the targeted host infection vulnerability (S) genes.

The pathogen formulate and translocates its virulence proteins, such as TAL effectors into the host cells, once internalized in the host cell TAL effectors binds to the promoter elements [effector-binding elements (EBEs)] of $\mathrm{S}$ genes, activating the expression of $\mathrm{S}$ gene which in turn develops more susceptible reaction between the host plants and bacterial pathogen. By making small changes at the specific TAL-effector binding site in the $\mathrm{S}$ genes, might lead the plants to be resistant to the bacterial pathogens, because TAL effector no longer would be capable to identify the target site of the S gene. Similarly, Targeted Gene-Editing in Rice has been carried out through employing CRISPR/Cas9 System. It's also substantially cost-effective, precise, user-friendly, and highly adapted for numerous gene targeting and highperformance genome-wide editing with a comparable or rather superior effectiveness than ZFNs and TALENs. 


\section{Aroma in rice}

The primary fragrance compound in the aromatic rice is 2-acetyl-1-pyrroline (2AP) which gets synthesised due to presence of non-functional betaine aldehyde dehydrogenase 2. BADH2 inhibits 2AP synthesis by diverting gama aminobutyraldehyed which is upstream precursor of gama amino butyric acid, by disrupting BADH2 gama-amino butyraldehyde is converted in acetyl pyrroline, consequently inducing fragrance in rice.

It has been observed that the disruption of BADH2 by using TALEN methodology increased 2AP content in grains from 0 to $0.35-0.75 \mathrm{mg} / \mathrm{kg}$, which was analogous to its documented content in the positive control aromatic variety (Shan et al., 2015).

\section{Powdery mildew resistant wheat}

Powdery mildew caused by an obligate biotrophic fangus, Blumeria graminis f.sp.tritici, which it's known as the most severe wheat crop disease, involves in drastic reduction of yield particularly in temperate zones. MLO locus being the target site of pathogen that encodes a G-protein, which through reverse adjustment the functionality of plant defence mechanisms, act as facilitator for the pathogen, orthologous MLO genes are ubiquitous among all higher plants. In case of mutant MLO disease inducing property is lost due to which mildew spreading is impeded from penetration to the cell wall or at the time of entry of host. Prof Caixia Gao and her team at the Chinese Academy of Sciences Institute of Genetics and Developmental Biology in Beijing, China, used TALEN (transcription activator-like effector nuclease) methodology, to successfully delete function of MLO genes. It was observed that the omozygous tamloaabbdd plants showed significant resistance to the powdery mildew infection.

\section{Declining of phytic acid in maize}

Maize kernels enriched with phosphorus, however, the large part of the $75 \%$ phosphorous stored as phytic acid which is not digestible by human. In addition, phytic acid is an anti-nutriental compound that negatively confine nutritional intake, farther, causes harmful impact on environment through inducing the waste stream. By using genome engineering procedures, phytic acid concentration can be reduced. In 2009, Shukla et al., engineered a ZFN create to modify the IPK1 gene, one of the genes that is responsible to regulate the biogenesis of phytic acid.

\section{Acrylamide-free potatoes}

Potatoes after harvesting are stored in cold chambers to enhance their shelf life, however during cold storage, starch in potatoes is degraded, thus when potatoes in frying process, at high temperatures the cold induced sugars converts to a brownish colour, at last, at the end of the process a strong poisonous acrylamide is formed. Voytas and his colleagues have designed TALEN constructs to alter VASCULAR INVERTASE genes, which by their functions, sucrose content of a potato can be transformed to glucose and fructose, they demonstrated that genomeedited potatoes after cold storage, then in at high temperatures, generated notably less brownish pigments and acrylamides as compared to wild-type potatoes (Clasen et al., 2015).

\section{Non-browning apples}

Genome editing techniques can be implemented to target polyphenol oxidases (PPO) genes in apple which are responsible for inducing brown colour in fresh cut apples, by mutating PPO genes their browning inducing activity can be declined. 
Fig.1 Mechanism of genome editing

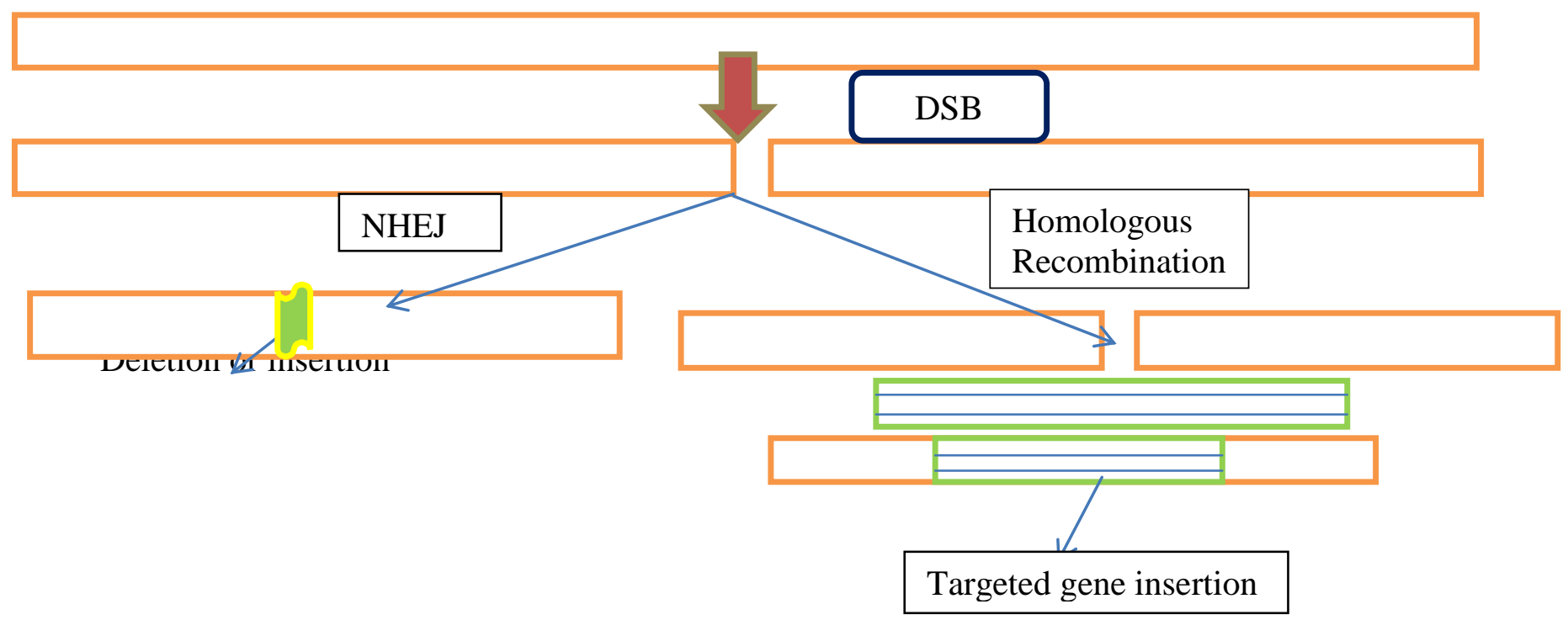

Table.1 Comparison between various genome editing tools

\begin{tabular}{|l|l|l|l|l|}
\hline & Mega nucleases & ZFN & TALENS & CRISPR/Cas9 \\
\hline $\begin{array}{l}\text { Recognition } \\
\text { site }\end{array}$ & $\begin{array}{l}\text { Between 12 and } \\
40 \mathrm{bp}\end{array}$ & $9-18 \mathrm{bp}$ & $14-20 \mathrm{bp}$ & $22 \mathrm{bp}$ \\
\hline Efficiency & Low & Medium & Medium & High \\
\hline $\begin{array}{l}\text { Off target } \\
\text { effects }\end{array}$ & More & More & Limited & $\begin{array}{l}\text { No off target } \\
\text { effect }\end{array}$ \\
\hline Construction & Highly Difficult & Highly Difficult & Difficult & Easy \\
\hline $\begin{array}{l}\text { Target DNA } \\
\text { recognition }\end{array}$ & Protein guided. & Protein guided & Protein guided & $\begin{array}{l}\text { RNA/DNA } \\
\text { hybrid. }\end{array}$ \\
\hline
\end{tabular}

Thus lead to produce apples which remain fresh for several weeks after they have been sliced into pieces.

\section{Regulation of ripening in tomato}

Ripening in tomato is regulated by RIN genes which in turn are encoded by MADS-box transcription factor. To target these regions within the gene, CRISPR/Cas9 system has been utilized, it was seen that homozygous RIN mutant tomato plants remained partially unripen, in contrast with the wild-type, in fact it's verifying the critical role of RIN in the maturation of tomato (Ito et al., 2015).

\section{Increased oleic acid level in soybean oil}

TALENs have been applied to scale down the activity of the two fatty acids desaturase genes in soybean, including FAD2 and FAD3, which converts monosaturated oleic acid to polyunsaturated linolenic acid to create plants which their seeds are contain instinctive amount of monosaturated oleic acid ( $80 \%$ vs. the normal $\sim 20 \%$ ) and low level of polyunsaturated fatty acid as well as linoleic acid ( $4 \%$ vs. the usual 50\%) (Haun et al., 2014), therefore, produces a healthier and high quality oil with an improved shelf life. 


\section{Herbicide-resistant crops}

Genome editing techniques has provided great success in generating herbicide tolerant crops. ZFN mediated genome editing induces a point mutation at a specific locus in the ACETOLACTATE SYNTHASE (ALS) gene, which is mainly targeted by herbicides, sulfonylurea (SU) and imidazolinone (IMI) (Townsend et al., 2009). These point mutation induced at the target site by genome editing can be useful in generating herbicide-resistant crops in future as it provides more efficient and accurate way as replacement of transgenic breeding.

\section{Coffee without caffeine}

Finding caffeine-free coffee has been coffee breeders goal for years (Borrell 2012), as the caffeine is highly toxic to humans, further processes need to be operated to eliminate caffeine from raw coffee bean which indeed it's a challenging and tedious processes, even sometimes it may generate harmful byproducts, and somehow lead to lessen or take away other aromatic compounds. In 2003, Ogita et al., studied RNAi constructs to silence the responsible gene for biogenesis caffeine, XANTHOSINE METHYL TRANSFERASE in Coffea canephora, known as Rubusta coffee. By practicing genome editing techniques other caffeine biosynthetic genes or caffeine transporters can be targeted in the future, as scientists trying to make coffee with very little if not without caffeine.

\section{Cotton}

The tangibility of targeted gene stacking in cotton by means of adopting specifically engineered mega nucleases has been reported (D'Halluin et al., 2013). In these experiments, gene present in the embryogenic cells of cotton possessing a site adjoining to an insect persistence has been targeted to promote double stranded breaks via homologous recombination in the presence of DNA template possessing two different genes for herbicide resistance flanked by DNA arrays with homology to the target site. Roughly2\% of individually modulated callus lines was indicated to consist no only the precise insertion but also to pass down the stacked features to the subsequent off springs.

\section{Herbicidal resistance in tobacco}

Mutation in two genes of tobacco i.e. SuRA and SuRB by adopting zinc finger nucleases has created herbicide resistant crops possessing resistance against imidazolinone and sulfonylurea.

\section{Canola}

Zinc finger protein have been used to alter the oil content in canola seeds by decreasing palmitic acid and increasing total C18 fatty acids which is done by enhancing the multiplication of the two canola b-ketoacylACP synthase II (KASII) genes in which the VP16 transcriptional activator domain had been connected (Gupta et al., 2012). Such studies have indicated that the engineered zinc finger protein transcription factors (ZFPTFs) can be exerted to adjust genome organisation in the main food plants and to obtain advantageous modifications to ameliorate the agronomically as well as economically important properties.

\section{Biosafety regulations}

Genome editing tools such as ZFNs, TALENs and CRISPR/Cas9 systems are the nova techniques employed to accurate mutate in myriad plant genomes with avoiding of entering an infinitesimal or no alien DNA. Woo et al., (2015) determined that naturally occurring mutations-like can be achieved in the genome editing by avoiding form 
introducing any exterior DNA with the aim of preassembled CRISPR/Cas9 proteins. Kanchiswamy (2016) previously detailed capabilities and significances of DNA-free genome editing in crops. Weather genome edited plants should be considered and were to put under the resemble regulation to GMOs or not, propelled scientific communities and legislators to have an ongoing argument on this matter. USDA in 2012 presented that ZFN-edited plants with no transgene interjection should not be treated with the established regulations for GMOs. Likewise in EU, genome edited crops mediated by ZFN are evaluated under European Community regulations. Further, the New Zealand Environmental Protection Authority (EPA) committee declared that ZFN-1 and TALENmediated engineered plants are not considered as GMOs (The Mc Guinness Institute 2013). Recently, Swedish Board of Agriculture reported that based on EU definition of GMO, CRISPR-Cas9 cannot be categorized as genetically modified plants. Lastly, USDA established no specific regulatory to cultivation as well as of CRISPR edited mushroom (Waltz 2016).

Agriculture has achieved great success during the last decade, however, as result of population explosion, there are constant demands while scientists are trying best to develop new and more efficient technologies that could help them to have proper responses to these vital requirements which the present and the future of mankind depends on..From production breeding breeders had shifted to resistance breeding than to resilience breeding than to genetic engineering approaches and mutation breeding. As the mutation is a random process and genetic engineering approaches are deal with growing number of regulations and social responsibility, due to biosafety regulations scientists are these days mostly concentrated on genome editing as this technique targets caused less specific disposition in genome, then low monitoring and crossing is required, further these new gene editing technologies are markedly, accurate, efficient, reliable, economy and user-friendly. Various genome editing tools have been utilized which among them CRISPR/Cas9 technique is the most efficient one and has abled scientists to create new opportunities for crop improvement, which capable of providing ground-breaking solutions for the food crisis. This is expected that the improved crops through genome editing techniques to be generally more acceptable for consumers as they legally registered as non-transgenic plants. Applying of genome editing techniques lawfully, beyond doubt supply plethora of food to deteriorate famine in the world, and interestingly their production will be in a sustainable manner in the close future.

\section{References}

Becker, M. 2012. Method of the year 2011. Nat Methods. doi:10.1038/nmeth.1852.

Borrell B. 2012. Plant biotechnology: Make it a decaf. Nat. News 483: 264-266.

Carroll, D. Genome engineering with targetable nucleases. 2014. Annu Rev Biochem. 83:40939; http://dx.doi.org/10.1146/annurevbiochem060713-035418.

Clasen, B.M., Stoddard, T.J., Luo, S., Demorest, Z.L., Li, J., Cedrone, F., Tibebu, R., Davison, S., Ray, E.E., Daulhac, A. 2015. Improving cold storage and processing traits in potato through targeted gene knockout. Plant Biotechnol. (in press).

D'Halluin K, Chantal V, Jolien VH, Joanna R, Ilse VDB, Anouk P, et al., Targeted molecular trait stacking in cotton through targeted double-strand break induction. Plant Biotechnol J. 2013; 11(8): 933-41.

Gaj, T., Gersbach, C.A., Barbas, C.F. 2013. ZFN, TALEN, and CRISPR/Cas-based methods for genome engineering. Trends Biotechnol. 31: 397405; http:// dx.doi.org/10.1016/j.tibtech. 2013.04.004.

Gallagher, R.R., Li. Z., Lewis, A.O., Isaacs, F.J. 2014. Rapid editing and evolution of bacterial 
genomes using libraries of synthetic DNA. Nature Protocols. 9(10); 2301-16.

Gilbert, L.A., Horlbeck, M.A., Adamson, B., Villalta, J.E., Chen, Y., Whitehead, E.H., et al., (2015). Genome-scale CRISPR-mediated control of gene repression and activation. Cell 159, 647-661. doi:10.1016/j.cell.2014.09.029

Gilbert, L.A., Larson, M.H., Morsut, L., Liu, Z., Brar, G.A., Torres, S.E., et al., (2013). CRISPR mediated modular RNA-guided regulation of transcription in eukaryotes. Cell 154, 442-451. doi:10.1016/j.cell.2013.06.044

Gupta M, DeKelver RC, Palta A, Clifford C, Gopalan S, Miller JC, Novak S, Desloover D et al., (2012) Transcriptional activation of Brassica napus beta-ketoacyl-ACP synthase II with an engineered zinc finger protein transcription factor. Plant Biotech J10:783-791

Haun W, Coffman A, Clasen BM, Demorest ZL, Lowy A, Ray E, et al., Improved soybean oil quality by targeted mutagenesis of the fatty acid desaturase 2 gene family. Plant Biotechnol J. 2014; 12(7): 934-40.

Ito, Y., Nishizawa, Yokoi, A., Endo, M., Mikami, M., Toki, S. 2015. CRISPR/Cas9-mediated mutagenesis of the RINlocus that regulates tomato fruit ripening. Biochem Biophys Res Commun. doi: 10.1016/ j.bbrc.2015.09.117.

Kanchiswamy, C.N. 2016. DNA-freegenome editing methods for targeted crop improvement. Plant Cell Rep. doi:10. 1007/s00299016-1982-2.

Kim, H., Kim, J.S. 2014. A guide to genome engineering with programmable nucleases. Nat. Rev. Genet. 15: 321-34.

Larson, M.H., Gilbert, L.A., Wang, X., Lim, W.A., Weissman, J.S., and Qi, L.S. (2013). CRISPR interference (CRISPRi) for sequencespecific control of gene expression. Nat.Protoc. 8, 2180-2196. doi:10.1038/nprot.2013.132.

Ogita, S., Uefuji, H., Yamaguchi, Y., Koizumi,
N., Sano, H. 2003. RNA interference: producing decaffeinated coffee plants. Nature. 423: 823-823.

Qi Y, Zhang Y, Zhang F, Baller JA, Cleland SC, Ryu Y, et al., Increasing frequencies of sitespecific mutagenesis and gene targeting in Arabidopsis by manipulating DNA repair pathways. Genome Res. 2013; 23(3): 547-54.

Shan, Q., Zhang, Y., Chen, K., Zhang, K., Gao, C. 2015. Creation of fragrant rice by targeted knockout of the OsBADH2 gene using TALEN technology. Plant Biotech J. 13:791800.

Shukla, V.K., Doyon, Y., Miller, J.C., DeKelver, R.C., Moehle, E.A., Worden, S.E., Mitchell, J.C., Arnold, N.L., Gopalan, S., Meng, X, et al., 2009. Precise genome modification in the crop species Zea mays using zinc-finger nucleases. Nature. 459: 437-441.

The Mc Guinness Institute. 2013. An Overview of Genetic Modification in New Zealand 19732013: The First Forty Years, Auckland Council. http:// www.auckland council.govt. $\mathrm{nz} / \mathrm{EN} /$ planspoliciesprojects/plansstrategies/uni taryplan/Documents/Section32report/Appendic es/Appendix\%203.49.16.pdf.

Townsend, J.A.Wright, D.A., Winfrey, R.J., Fu, F., Maeder, M.L., Joung, J.K., Voytas, D.F. 2009. High-frequency modification of plant genes using engineered zinc-finger nucleases. Nature. 459: 442-445.

Waltz,E.2016. Gene-edited CRISPR mushroom escapes US regulation. Nature. 532: 293. doi:10.1038/nature.2016.19754.

Woo, J.W., Kim, J., Kwon, S., Corvala, C., Cho, S.W., Kim, H., Kim, S., Kim, S., Choe, S., Kim, J. 2015. DNA-free genome editing in plants with preassembled CRISPR-Cas9 ribo nucleoproteins. Nat Biotechnol. 33: 11621164. doi:10.1038/nbt.3389.

\section{How to cite this article:}

Asma Majid, G.A. Parray, Shabir H. Wani, Mojtoba Kordostami, N.R. Sofi, Showkat A. Waza, A.B. Shikari and Shazia Gulzar. 2017. Genome Editing and its Necessity in Agriculture. Int.J.Curr.Microbiol.App.Sci. 6(11): 5435-5443. doi: https://doi.org/10.20546/ijcmas.2017.611.520 